\title{
The Effects of Irinotecan on the Healing of Colonic Anastomoses in Rats
}

\author{
Manousos-Georgios Pramateftakis ${ }^{*}$, , Dimitrios Kanellos ${ }^{1}$, Haralampos Demetriades ${ }^{1}$, \\ Ioannis Kanellos ${ }^{1}$, Ioannis Mantzoros ${ }^{1}$, Emmanouil Zacharakis ${ }^{1}$, Kalliopi Despoudi ${ }^{1}$, \\ Stamatios Angelopoulos ${ }^{1}$, Georgios Koliakos ${ }^{2}$, Thomas Zaraboukas ${ }^{3}$ and Dimitrios Betsis ${ }^{1}$ \\ ${ }^{1} 4$ th Surgical Department, Aristotle University of Thessaloniki, "G. Papanikolaou General Hospital, 57010 Exohi, \\ Thessaloniki, Greece \\ ${ }^{2}$ Biochemical Laboratory, Aristotle University of Thessaloniki, Greece \\ ${ }^{3}$ Histopathology Department, Aristotle University of Thessaloniki, Greece
}

\begin{abstract}
Aim: The aim of this experimental study was to investigate the effects of intraperitoneally injected irinotecan on the healing of colonic anastomoses after colon resection.

Methods: Thirty male Wistar rats were used. The rats were randomized into two groups of 15 rats each. Immediately after colonic anastomoses were performed, the rats were injected intraperitoneally with either $3 \mathrm{ml}$ of $0.9 \% \mathrm{NaCl}$ solution or irinotecan $(3 \mathrm{mg} / \mathrm{kg}$ body weight) depending on their group. All rats were sacrificed on the eighth postoperative day. The anastomoses were examined macroscopically and histologically. The anastomotic bursting pressures were recorded.
\end{abstract}

Results: Anastomotic dehiscence was noted in 3 rats of the irinotecan group. All anastomoses of the control group remained intact until sacrifice. The adhesion formation at the anastomotic sites and the average inflammatory cell infiltration scores were significantly higher in the irinotecan group compared to the control group. The bursting pressures, the hydroxyproline tissue content, the fibroblast activity and the collagen deposition were significantly lower in the irinotecan group. Neoangiogenesis did not differ significantly between the groups.

Conclusion: Irinotecan, when injected intraperitoneally, affects the healing of colonic anastomoses in rats.

Keywords: Irinotecan, colonic anastomosis, healing.

\section{INTRODUCTION}

It is well documented that after colon resection for cancer, the immediate, postoperative, intraperitoneal administration of cytostatica induces the destruction of cancer cells that have been disseminated during the procedure. Furthermore, administration of such drugs inhibits regional recurrence and liver metastases $[1,2]$. However, according to the results of previous experimental studies performed in our laboratory as well as those of other researchers, the immediate postoperative administration of cytostatica impairs wound healing of colonic anastomoses and results in a higher incidence of anastomotic failure after colon resection [3, 4]. Irinotecan is a new chemotherapeutic agent, recently introduced in the field of chemotherapy for colorectal cancer. Intraperitoneal injections of irinotecan have not been used in humans yet. So far, the effects of irinotecan on the healing of colonic anastomoses have not been assessed in detail [5].

The aim of this experimental study was to investigate the effect of irinotecan on colonic healing when injected intraperitoneally after colon resection.

\section{MATERIALS AND METHODOLOGY}

Thirty male Wistar rats, weighing between $120 \mathrm{~g}$ and $168 \mathrm{~g}$, were used. All animals received humane care in

*Address correspondence to this author at Antheon 1, Panorama 55236, Thessaloniki, Greece; Tel: 00302310314313; Fax: +30 2310 358000;

E-mail: mpramateftakis@hotmail.com accordance with the "Principles of Laboratory animal Care" formulated by the National Society for Medical Research and the "Guide for the Care and Use of Laboratory Animals" prepared by the Institute of Laboratory Animal Resources and published by the National Institute of Health (NIH Publication No. 86-23). Principles of laboratory animal care were followed and the research protocol described was approved by the Ethical Committee of the Department of Veterinary Services of the Prefecture of Thessalonica according to the Greek Law on the protection of animals. Animals were housed individually and had unrestricted access to the standard laboratory diet and water pre- and post-operatively.

\section{Anaesthesia and Operative Technique}

The rats were weighed on the day of the operation as well as before the sacrifice. Changes in weight were recorded. Surgery was performed through a $3-\mathrm{cm}$ midline incision under intraperitoneal thiopental anaesthesia $(50 \mathrm{mg} / \mathrm{kg}$ body weight). A $1 \mathrm{~cm}$ long segment was resected out of the middle transverse colon and an end-to-end anastomosis was performed with eight interrupted sutures (6/0 polypropylene). Mass closure of the abdominal muscle layer and the skin was performed using three sutures (3/0 silk).

\section{Groups}

During the operation the rats were randomized to one of two groups of 15 rats each. Randomization was through the use of computer-generated random numbers and there was allocation concealment after randomization. Immediately after the formation of the colonic anastomoses, the rats in the 
control group were injected with $3 \mathrm{ml}$ of $0.9 \% \mathrm{NaCl}$ solution intraperitoneally. Rats in the irinotecan group received irinotecan in a dose of $3 \mathrm{mg} / \mathrm{kg}$ body weight intraperitoneally.

\section{Macroscopic Assessment}

All rats were sacrificed on the eighth postoperative day. During post-mortem examination, macroscopic assessment of the anastomoses was performed; the integrity of the anastomosis, the presence of abscesses at the anastomotic sites, the formation of adhesions and any signs of generalised peritonitis were recorded. The results were evaluated in a blind fashion according to the scale of van der Hamm et al. [6] as follows: $0=$ no adhesions; $1=$ minimal adhesions, mainly between anastomosis and the omentum; 2 = moderate adhesions, between the omentum and the anastomotic site and between the anastomosis and a loop of the small bowel; and $3=$ severe and extensive adhesions, including abscess formation.

\section{Bursting Pressures}

During sacrifice, a 5-cm segment of the transverse colon, with the anastomosis at the centre and $2.5 \mathrm{~cm}$ of colon on either side, was carefully removed along with the adhesions that had been formed. Faeces were washed out of this segment and the proximal end was ligated with the use of a $4 / 0$ silk suture. A catheter, which was connected to a sphygmomanometer, was inserted into the distal end of the lumen and the bowel was firmly ligated around the tube. Through this catheter, a $0.9 \% \mathrm{NaCl}$ solution was infused at a constant rate of $1 \mathrm{ml} / \mathrm{min}$. The bursting pressure, meaning the pressure at which any leakage of saline or gross rupture of the bowel segment was noted, was recorded in $\mathrm{mmHg}$. The specific area of the leakage or rupture was also recorded, being at the anastomotic site or far from it. All bursting pressure measurements were obtained immediately after sacrifice.

\section{Histological Assessment}

Once the bursting pressure was recorded, the anastomosic site was resected along with a $0.5-\mathrm{cm}$ distal and proximal portion of the colon. The anastomosis was incised longitudinally and divided into two equal segments. The first segment was placed in $4 \%$ formalin solution for histological assessment. After being stained with hematoxylin and eosin, the anastomosis was graded histologically in a blind fashion, using the Ehrlich and Hunt numerical scale from 0 to 4 as modified by Phillips et al. [7]. Inflammatory cell infiltration (white blood cell count), fibroblast activity, new blood vessel development (neoangiogenesis) and collagen deposition were graded from 0 to 4 as follows: $0=$ no evidence, $1=$ occasional evidence, 2 = light scattering, $3=$ abundant evidence, and $4=$ confluent cells or fibres. All histological assessments were carried out by an experienced histopathologist who was blinded.

\section{Hydroxyproline}

The second segment of the anastomosis was weighed and stored at $-70^{\circ} \mathrm{C}$ for measurement of hydroxyproline. The process of determination of hydroxyproline tissue contents is described in detail in relevant literature [8]. Briefly, after the specimens were lyophilized, a polytron homogenizer was used to homogenize the tissue sample in distilled water. In addition to this fine tissue homogenate, purified acid-soluble collagen (dissolved in $50 \mathrm{mM}$ acetate buffer, $\mathrm{pH}$ 3.5) was included as a test sample for the estimation of hydroxyproline. Aliquots of standard hydroxyproline/test samples were hydrolyzed in alkali. The hydrolyzed samples were then mixed with a buffered chloramines-T reagent $(0.056 \mathrm{M})$, and the oxidation was allowed to proceed for $25 \mathrm{~min}$ at room temperature. Ehrlich's reagent (1M) was used to develop the chromophore. The absorbance of reddish purple complex was measured at $550 \mathrm{~nm}$ using a Gilford Response ${ }^{\mathrm{TM}}$ spectrophotometer. Absorbance values were plotted against the concentration of standard hydroxyproline, and the presence of hydroxyproline in unknown tissue extracts was determined from the standard curve.

\section{Statistical Analysis}

Descriptive statistics for discrete variables were presented as frequencies and percentages. Continuous variables were summarized using means with $95 \%$ confidence intervals and medians with ranges from minimum to maximum. The statistical method employed was Fisher's exact test for comparison of proportions. Differences among groups, with respect to non-normally distributed adhesions, weight changes, bursting pressures, hydroxyproline levels and the wound healing process were tested using the Kruskal-Wallis test, whereas pair-wise differences were compared by the Mann-Whitney test, at a Bonferroni-adjusted significance level. All analyses were conducted using SPSS 12 (SPSS, Inc., Chicago, IL). All reported p-values were two-tailed with $p<0.05$ considered as significant.

\section{RESULTS}

Anastomotic dehiscence was noted in 3 rats (20\%) of the irinotecan group, whereas all anastomoses of the control group remained intact up to the day of the sacrifice. Application of Fisher's exact test for the control group versus irinotecan group showed no statistical relevance between group and anastomotic dehiscence $(\mathrm{p}=0.111)$.

Statistically significant postoperative decrease of the rats' body weight was noted in both groups. A $9 \%$ body weight decrease was noted in the irinotecan group (Wilcoxon test: $\mathrm{p}=0.001$ ), with the mean body weight decreasing from $153.47 \mathrm{~g}$ before the experiment to $140.33 \mathrm{~g}$ before sacrifice. In the control group, the mean body weight decreased from $153.93 \mathrm{~g}$ before the experiment to151.33g before sacrifice, resulting to a $2 \%$ body weight decrease (Wilcoxon test: $\mathrm{p}=0.002$ ). A comparison of the two groups revealed that the weight decrease in the irinotecan group was statistically significantly higher than in the control group (Mann-Whitney: $\mathrm{p}<0.001$ ). The body weight changes are presented in Fig. (1).

\section{Adhesion Formation}

The adhesion formation was significantly higher in the irinotecan group (mean 1.93) than in the control group (mean 0.73). (Fig. (2), Mann-Whitney: $\mathrm{p}<0.001$ ).

\section{Bursting Pressure}

The bursting pressures were significantly lower in the irinotecan group (mean $174,5 \mathrm{mmHg}$ ) compared to the control group (mean 270,87 mmHg) (Table 1, Mann-Whitney: $\mathrm{p}<0.001) .3$ anastomoses of the irinotecan group dehisced before sacrifice. Their bursting pressures were valued as 
zero. These insufficient anastomoses were not included in the calculations of the bursting pressure analysis.

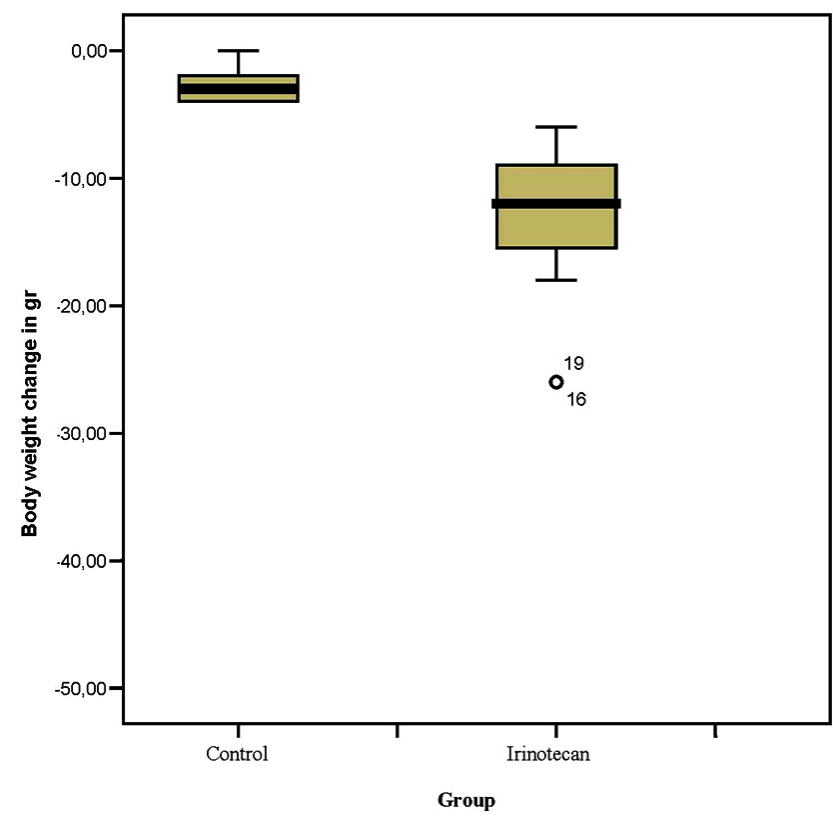

Fig. (1). Body weight change in grams (gr) in the two groups.

Plotted are means with standard errors. Overall comparison with Kruskal-Wallis test, $\mathrm{p}<0.001$. Using the Mann-Whitney test and the Bonferroni adjustment: control $v s$ irinotecan, 2.6 vs 13.14, $\mathrm{p}<0.001$.

In Figs. (1-3), represented are the median and the range. The numbers at the asterisk and the small circles represent the amount of animals whose values lie outside the range. The bottom edge of the box represents $25 \%$ of the measurements, whereas $75 \%$ of the measurements are represented by the top edge.

\section{Histological Assessment}

The histological assessment of the anastomotic healing included measurements of inflammatory cell infiltration, new vessel formation, collagen deposition and fibroblast activity. The average inflammatory cell infiltration was significantly higher in the irinotecan group (mean 2.67) than the control group (mean 1.2, Kruskal-Wallis: $\mathrm{p}<0.001$ ). The inflammatory cell infiltration results are presented in Fig. (3). Neoangiogenesis was similar between the two groups (mean 1.6, Kruskal-Wallis, $\mathrm{p}>0.2$ ). Both the average collagen deposition score (mean 0.6 vs 2.87, Kruskal-Wallis: $\mathrm{p}<0.001$ ) and the fibroblast activity (mean 1.6 vs 3.47, Kruskal-Wallis: $\mathrm{p}<0.001)$ were significantly lower in the irinotecan group than the control group. Signs of intestinal damage such as mucositis were not reported by the histopathologist during the assessment.

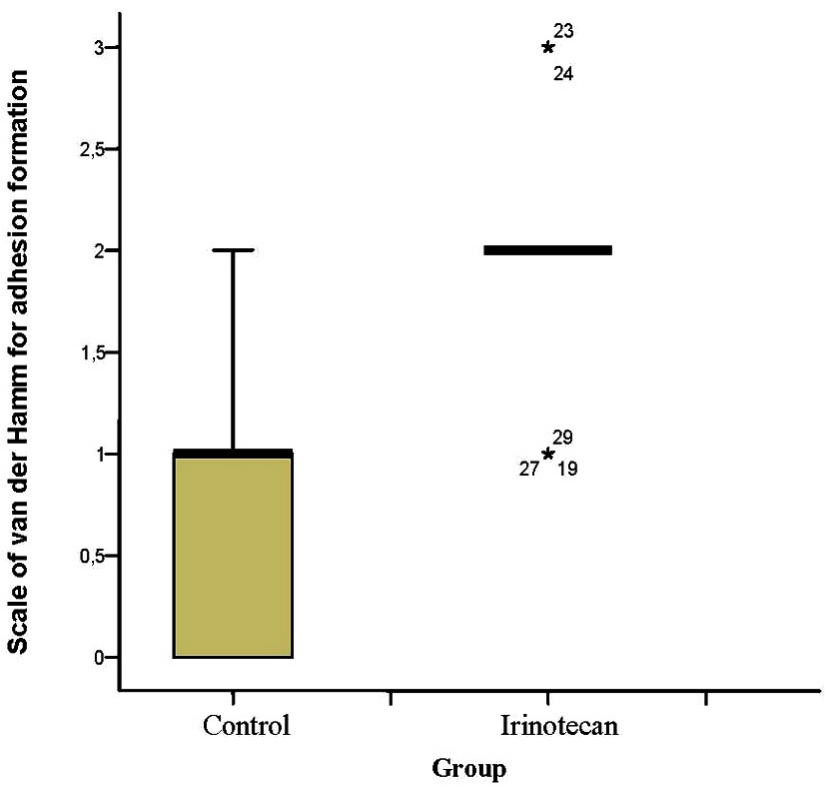

Fig. (2). Formation of adhesions according to the scale of van der Hamm (0-3).

Plotted are means with standard errors. Overall comparison with Kruskall-Wallis test, $\mathrm{p}<0.001$. Using the Mann-Whitney test and the Bonferroni adjustment: control vs irinotecan, 0.73 vs 1.93, $\mathrm{p}<0.001$.

\section{Hydroxyproline}

There was also a statistically significant decrease of the hydroxyproline tissue content of the irinotecan group compared to the control group (Mean 1.2960 vs 3.2213, MannWhitney: $p<0.001$. The results for the hydroxyproline tissue contents are presented in Table 2.

\section{DISCUSSION}

Since the arrival of 5-fluorouracil (5-FU) in the treatment of colorectal cancer approximately 50 years ago, alterations in the use and administration of 5-FU have only improved the median survival of patients from 6-9 months to 12 months [9]. Nowadays, the combination of 5-FU with newer drugs that have no cross-resistance with 5-FU, such as irinotecan and oxaliplatin, seems to improve patients' response rates and provide better survival benefits. A multicenter trial by Fuchs et al. [10] in 2006 has shown that the combination of irinotecan with 5-FU/Leucovorin as a first-line treatment for metastatic colorectal cancer can significantly prolong survival compared to 5-FU/Leucovorin alone. The same study reported that irinotecan-based regimens may improve resectability of metastases and further improve patient survival. Irinotecan has also been used in concurrence with oxaliplatin and UFT in first-line treatment regimens in an at-

Table 1. Descriptive Values of Bursting Pressures (mmHg) in the Two Groups

\begin{tabular}{|c|c|c|c|c|c|c|}
\hline Group & $\mathbf{n}^{\mathrm{a}}$ & Mean & 95\% C.I. & Median & Range & p-Value ${ }^{b}$ \\
\hline Control & 15 & 270.87 & $253.45-288,28$ & 276 & $218-314$ & \multirow{2}{*}{$<0.001$} \\
\hline Irinotecan & 12 & 174.5 & $153.97-195.03$ & 168 & $120-228$ & \\
\hline
\end{tabular}

C.I.: Confidence interval.

3 anastomoses of the irinotecan group dehisced before sacrifice. Their bursting pressures were valued as zero and were not included in the descriptive analysis of this table.

${ }^{\mathrm{b}}$ Mann-Whitney test with Bonferroni adjustment. 
tempt to reduce the drug's dose-limiting side effects. Sheikh et al. [11] have shown that alternating irinotecan and oxaliplatin plus UFT is a well-tolerated first-line treatment for patients with advanced colorectal cancer. Furthermore, studies are being conducted on the concurrent administration of irinotecan and radiation for patients with locally advanced rectal cancer [12].

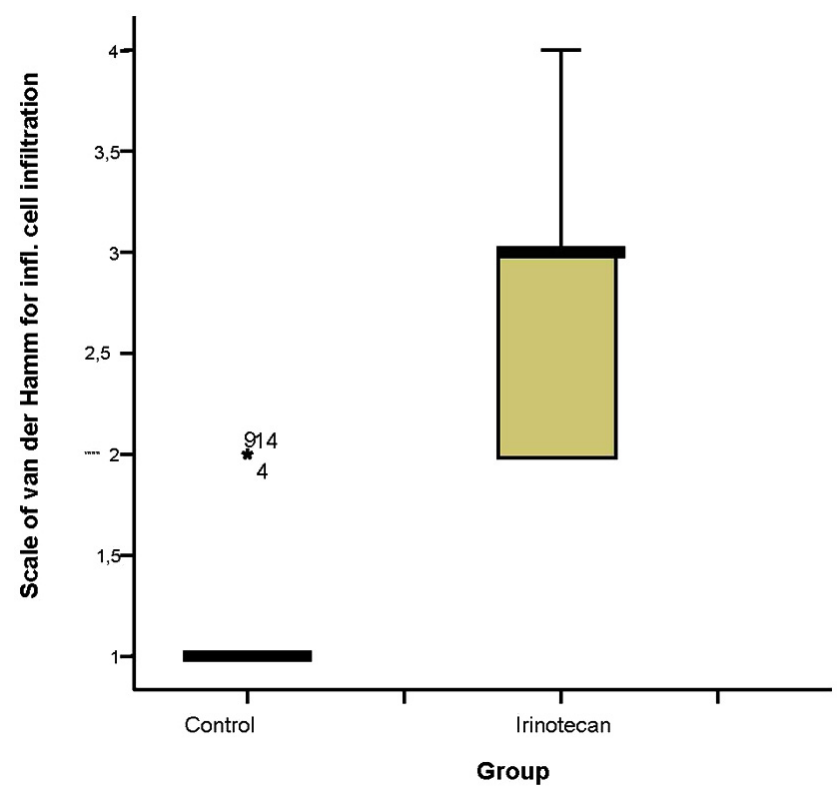

Fig. (3). Inflammatory cell infiltration according to the scale of van der Hamm (0-3).

Plotted are means with standard errors. Overall comparison with Kruskall-Wallis test, $\mathrm{p}<0.001$. Using the Mann-Whitney test and the Bonferroni adjustment: control vs irinotecan, 1.2 vs 2.67, $\mathrm{p}<0.001$.

The healing process of colonic anastomoses has been shown to be inhibited by various factors that can be divided in general and topical ones. General factors include the patient's age, nutritional status, medication and chronic diseases such as hepatic impairment and diabetes [13-16]. Ischemia and hypoxia at the anastomotic site, usually due to the formation of anastomoses under tension, are the most common topical factors impairing anastomotic healing [17].

The protection of anastomoses during that vulnerable healing period is an important task in the immediate postoperative time. As mentioned before, malnutrition is one of the main general impairment factors of the healing process, partly because of the reduced collagenization at the anastomotic site [18]. Early postoperative nutrition improves the anastomotic healing and the oral administration of elementary diet enriched with arginine and glutamine increases the collagen synthesis at the anastomosis [19]. Experiments by Tingstedt et al. [20] on a rat model have shown that bioac- tive polypeptides like poly-L-lysine and poly-L-glutamate, when administered intraperitoneally, reduce the formation of abdominal adhesions and increase the anastomotic strength. The latter was shown via an increase in the anastomotic bursting pressures.

The effects of different chemotherapeutic agents on the healing process of anastomoses have been studied by various researchers. Kanellos et al. [21] studied the effects of 5-FU on the anastomotic healing in a rat model and concluded that 5-FU impairs the healing process. Additional intraperitoneal injections of interferon or folic acid did neither improve nor worsen the results [3]. Haciyandli et al. [22] studied the effects of intravenously or intraperitoneally administered leucovorin in conjunction with 5-FU on anastomoses. They concluded that there were no further positive or negative effects on anastomotic healing or on the impairment caused by 5-FU. In 2007, Kostantinidis et al. [23] assessed the effects of Capecitabine, a selective pro-drug of 5-FU, on the healing of colonic anastomoses. Capecitabine, when given perioperatively, was not found to have a negative effect on anastomotic healing.

To our knowledge, there are no studies up-to-date assessing the effects of irinotecan on the process of anastomotic healing. Irinotecan, also known as CPT-11, is a topoisomerase I inhibitor with established activity in first- and second-line treatment regimens of colorectal cancer [10]. It is a derivate of camptothecine, a natural alkaloid isolated from the Chinese tree Camptotheca accuminata. It blocks the DNA replication mediated by topoisomerase I and induces breaks in the single strand DNA, an action which inhibits DNA replication [24].

Colonic anastomotic dehiscence is a much feared complication in the immediate postoperative period of colonic resections. Such leakages significantly increase the rates of morbidity and mortality after large bowel resections [25]. The rates of dehiscence in experimental studies on animal models vary between $3 \%$ and $30 \%$ [25-28]. In our study, $20 \%$ of the irinotecan group anastomoses dehisced before sacrifice. There was no statistically significant difference in terms of the anastomotic dehiscence between the two groups.

Cancer patients undergoing chemotherapy usually suffer weight loss. That weight loss is usually due to the sideeffects of chemotherapy. Nausea and anorexia, main side effects of different chemotherapeutic agents, usually lead to decreased appetite and weight loss. Intraperitoneal steroids in a study in a rat model have been shown to decrease postoperative weight 7 days after administration [29]. 5-FU has also been shown to decrease weight following intraperitoneal administration in rats [21]. In our study, there was statistically significant weight loss following intraperitoneal administration of irinotecan.

Table 2. Descriptive Values of Hydroxyproline Tissue Contents $(\mu \mathrm{g} / \mathrm{mg})$ in the Anastomoses of the Two Groups

\begin{tabular}{|c|c|c|c|c|c|}
\hline Group & $\mathbf{n}$ & Mean & $\mathbf{9 5 \%}$ C.I. & Median & p-Value \\
\hline \hline Control & 15 & 3.2213 & $1.136-1.41$ & 3.18 & 1.28 \\
\hline Irinotecan & 15 & 1.2960 & $1.1526-1.43$ & $<0.001^{\text {a }}$ \\
\hline
\end{tabular}


The mechanical strength of the anastomoses is determined by the bursting pressure, the pressure at which the anastomosis leaks. The bursting pressure increases progressively after the formation of the anastomosis. It is therefore a useful parameter to measure the healing process during the first week after the anastomotic formation [30]. In our study, the bursting pressures were significantly lower after the administration of irinotecan.

Anastomotic healing is a complex process. The regeneration of damaged tissue includes the interaction of many cellular components and it can be affected by many different parameters. The process of healing is divided into four different phases [31].

Inflammatory cell infiltration takes place during the second phase of wound healing, the inflammatory phase. Inflammatory cells, guided by the presence of chemotactic factors, reach the anastomotic site a few minutes after the bowel resection. Their number maximises between the first and second postoperative day [32]. Chemotherapeutic agents have been shown to increase inflammatory cell infiltration in tumour tissue [33]. In our study there was statistically significant increase of inflammatory cell infiltration following intraperitoneal administration of irinotecan compared to the control group.

Neoangiogenesis, the formation of new vessels from the endothelium of pre-existing vessels, is an important element of the healing process, finding place in its third phase, the productive phase. It has been shown to be affected by various angiogenic factors. Studies by Rabinovsky et al. [34] on a mouse diabetic model have shown that Insulin-like growth factor I, an anabolic agent that mediates body weight through protein and fat metabolism, promotes neovessel formation. In our study, the degree of neoangiogenesis has not been affected by the presence of intraperitoneal irinotecan.

Fibroblast activity, leading to collagen deposition, occurs mainly during the third phase of the healing process. Fibroblasts migrate to the anastomotic site from surrounding tissues and start the collagen production. Collagen is the main protein of connective tissue in animals and is made up of three polypeptide strands, forming a triple helix. The stability of that helix is maintained with the assistance of various factors. One of the main factors is hydroxyproline, an amino acid. It permits the sharp twisting of the collagen helix and helps to provide stability by forming hydrogen bonds. The fact that it is only found in a few proteins other than collagen has made it an important indicator of collagen amount determination. Many cytostatics have been shown to reduce fibroblast activity, as part of their cytotoxic effects. A study by Bulstrode et al. [35] has shown that 5-FU, which has been used for the treatment of fibroproliferative disorders of the skin and the eye, selectively inhibits collagen synthesis. In our study, both fibroblast activity and collagen deposition were significantly decreased in the irinotecan group compared to the control group. The hydroxyproline tissue content was also significantly lower in the irinotecan group compared to the control group.

\section{CONCLUSION}

In conclusion, irinotecan, when given intraperitoneally, affects the healing of colonic anastomoses in rats. Further studies are required to assess interactions of irinotecan with other drugs and any dose-related changes to its effects.

\section{REFERENCES}

[1] Brodsky J, Cohen A. Peritoneal seeding following potentially curative resection of colonic carcinoma: Implications for adjuvant therapy. Dis Colon Rectum 1991; 34: 723-727.

[2] Sugarbaker P, Gianola F, Speyer J, Wesley R, Barofsky I, Meyers C. Prospective randomized trial of intravenous versus intraperitoneal 5-fluorouracil in patients with advanced primary colon or rectal cancer. Surgery $1985 ; 98: 414-420$.

[3] Kanellos I, Kavouni A, Zaraboukas T, Odisseos C, Galovatsea K, Dadoukis I. Influence of intraperitoneal 5-fluorouracil plus folinic acid on the healing of colonic anastomoses in rats. Eur Surg Res 1996; 28: 374-379.

[4] Weiber S, Graf W, Glimelius B, Jiborn H, Pahlman L, Zeberfelbt B. Experimental colonic healing in relation to timing of 5fluorouracil therapy. Br J Surg 1994; 81: 1677-1680.

[5] Ajani JA, Faust J, Yao J, et al. Irinotecan/Cisplatin followed by 5FU/paclitaxel/radiotherapy and surgery in oesophageal cancer. Oncology (Huntingt) 2003; 17(8 Suppl): 20.

[6] Van der Hamm A, Kort W, Weijma I, Van Der Ingh H, Jeekel H. Effect of antibiotics in fibrin sealant on healing colonic anastomosis in the rat. Br J Surg 1992; 74: 525-528.

[7] Philips JD, Kim CS, Fonkalsrud EW, Zeng H, Dindar H. Effects of chronic corticosteroid and vitamin A on the healing of intestinal anastomoses. Am J Surg 1992; 163: 71-77.

[8] Reddy GK, Enweneka CS. A simplified method for the analysis of hydroxyproline in biological tissues. Clin Biochem 1996; 29: 225229.

[9] Quinn MJ, Babb PJ, Brock A, Kirby EA, Jones J. Cancer trends in England \& Wales 1950-1999. 2001; Vol. SMPS No. 66: TSO.

[10] Fuchs C, Mitchell EP, Hoff PM. Irinotecan in the treatment of colorectal cancer. Cancer Treat Rev 2006; 32(7): 491-503.

[11] Sheikh HY, Valle JW, Palmer K, et al. Concurrent irinotecan, oxaliplatin and UFT in first-line treatment of metastatic colorectal cancer: a Phase I study. Br J Cancer 2007; 96: 38-43.

[12] Gill S, Blackstock AW, Goldberg RM. Colorectal Cancer. Mayo Clin Proc 2007; 82(1): 114-129.

[13] Kanellos I, Blouhos K, Demetriades H, et al. The failed intraperitoneal colon anastomosis after colon resection. Tech Coloproctol 2004; 8(Suppl 1): s53-5.

[14] Platel C, Barwood N, Dorfmann G, Makin G. The incidence of anastomotic leaks in patients undergoing colorectal surgery. Colorectal Dis 2007; 9(1): 71-79.

[15] Golub R, Golub RW, Cantu R Jr, Stein HD. A multivariate analysis of factors contributing to leakage of intestinal anastomoses. J Am Coll Surg 1997; 184(4): 364-72.

[16] Lipska MA, Bissett IP, Parry BR, Merrie AE. Anastomotic leakage after lower gastrointestinal anastomosis: men are at a higher risk. ANZ J Surg 2006; 76(7): 579-85.

[17] Amoruso M, D'Abbicco D, Notarnicola A, et al. Early colorectal reoperation: risk factors. Chir Ital 2001; 53: 827-33.

[18] Alamo JM, Galindo A, Morales S, et al. Role of malnutrition in intestinal anastomosis collagenization: an analysis of procollagen (PINP) and carboxyterminal telopeptide (ICTP) by radioimmunoassay. Rev Esp Enferm Dig 2007; 99(2): 76-83.

[19] Da Costa MA, Campos AC, Coelho JC, de Barros AM, Matsumoto HM. Oral glutamine and the healing of colonic anastomoses in rats. JPEN J Parenter Enteral Nutr 2003; 27: 182-5.

[20] Tingstedt B, Nehez L, Lindman B, Andersson R. Effect of bioactive polypeptides on leaking large bowel anastomosis and instestines in the rat. J Invest Surg 2007; 20(4): 229-35.

[21] Kanellos I, Odisseos C, Zaraboukas T, Kavouni A, Galovatsea K, Dadoukis I. Colonic healing after early intraperitoneal administration of 5-fluorouracil and interferon in rats. Int $\mathrm{J}$ Colorectal Dis 1997; 12(1): 45-8.

[22] Haciyandli M, Fuzun M, Unek T, Tokgoz Z. Does the administration route of Leucovorin have any influence on the impairment of colonic healing caused by intraperitoneal 5-fluorouracil treatment? Eur Surg Res 2001; 33(2): 80-5.

[23] Konstantinidis HD, Slavakis AP, Ballas KD, et al. The effect of capecitabine on the healing of colonic anastomoses in rats. Dis Colon Rectum 2007; 50(1): 89-96. 
[24] Wang JC. Cellular roles of DNA topoisomerases: a molecular perspective. Nat Rev Mol Cell Biol 2002; 3: 430.

[25] Demetriades H, Kanellos I, Vasiliadis K, et al. Age-associated prognosis following curative resection for colorectal cancer. Tech Coloproctol 2004; 8: 144-6.

[26] Cihan A, Oguz M, Acun Z, et al. Comparison of early postoperative enteral nutrients versus chow on colonic anastomotic healing in normal animals. Eur Surg Res 2004; 36: 112-5.

[27] Ehrlich HP, Hunt TK. The effects of cortisone and anabolic steroids on the tensile strength of healing wounds. Ann Surg 1969; 170: 203-6.

[28] Bitar M, Labbad Z. Transforming growth factor-b and insulin growth factor-I in relation to diabetes-induced impairment of wound healing. J Surg Res 1996; 61: 113-9.

[29] Mantzoros I, Kanellos I, Angelopoulos S, et al. The effect of insulin-like growth factor I on healing of colonic anastomoses in cortisone-treated rats. Dis Colon Rectum 2006; 49(9): 1431-8.
[30] Cihan A, Armutcu F, Ucan BH, et al. Comparison of the measurement methods of bursting pressure of intestinal anastomoses. Hepatogastroenterology 2003; 50: 232-4.

[31] Steed DL. Wound-healing trajectories. Surg Clin North Am 2003; 83: 547-55.

[32] Brunner G. Theme tissue: Wound healing mechanisms. Thromb Haemost 2003; 90: 976-7.

[33] Bovo G, Brivio F, Brenna A, et al. Pre-operative interleukin-2 immunotherapy induces eosinophilic infiltration in colorectal neoplastic stroma. Pathologica 1995; 87(2): 135-8.

[34] Rabinovsky ED, Draghia-Akli R. Insulin-like growth factor I plasmid therapy promotes in vivo angiogenesis. Mol Ther 2004; 9: 4655.

[35] Bulstrode NW, Mudera V, McGrouther DA, Grobbelaar AO, Cambrey AD. 5-fluorouracil selectively inhibits collagen synthesis. Plast Reconstr Surg 2005; 116(1): 209-21. 